\title{
POSSIBLE MUSCARINIC-CHOLINERGIC MEDIATION OF PATTERNED AGGRESSIVE REFLEXES IN THE CAT
}

\author{
R.J. KATZ \\ Mental Health Research Institute \\ Department of Psychiatry \\ University of Michigan Medical Center \\ Ann Arbor, MI USA
}

(Final form, November 1980)

\begin{abstract}
1. Adult female outbred cats were prepared with chronic hypothalamic stimulating electrodes and ventricular cannulae.

2. Cats were restrained, and patterned aggressive reflexes of elicited head turning, biting, and paw striking to proximate stimulation were assessed under a varlety of stimulation conditions.

3. In comparison with vehicle injection cholinergic stimulation by arecoline facilitated the three reflexes and cholinergic blockade by scopolanine inhibited them.

4. Cholinergic-muscarinic receptors may be involved as mediators of terminal (consummatory) behaviors in aggressive syndromes.
\end{abstract}

Key words: arecoline, aggression, cholinergic, lateral hypothalamus, muscarinic, predatory behavior, scopolamine.

\section{Introduction}

Flynn and colleagues (Flynn 1967, 1972) have demonstrated that electrical stimulation of diencephalic sites affects both sensory and motor aspects of aggression. So called "patterned reflexes" of biting and paw-striking are elicited in proportion to central and peripheral stimulation parameters, and, as has been demonstrated by Berntson and Micco (1976), the hierarchical sensory organization of these reflexes may be sufficient to subserve highly flexible goal-directed behaviors.

We have argued that muscarinic-cholinergic neurons may control critical aspects of both predatory (i.e. quiet biting) and affective (i.e. rage) aggression (Katz and Thomas, 1975; Katz, 1976). However, the specific involvement of acetylcholine in patterned reflexes has not yet been extensively studied. The present study therefore utilized cholinergic stimulation and blockade to further examine cholinergic influences upon the terminal aspects of attack (i.e. patterned reflexes). 


\section{Subjects}

The subject pool consisted of eight adult female cats weighing 2.0 to 3.5 kg, obtained from a local supplier (Bio Medical Assoc. Inc., Friedensburg, $P A$ ). All subjects were individually maintained on ad libitum food (Purina cat chow) and water throughout the experiment. Normal day/night cycles of 14 hours. daylight/10 hours darkness were maintained by natural and artificial lighting.

\section{Drugs}

Solutions of arecoline $\mathrm{HCl}$ (Sigma Biochemical A-4506) $1.7 \mathrm{mg}$ in 50 ul Ringer-Locke's solution were injected into the third ventricle 10 minutes prior to behavioral testing. To prevent confounding of central and peripheral cholinergic stimulant effects all arecoline injections were preceded by systemic injections of atropine methyl nitrate (Sigma Biochemicals A-0382) $1 \mathrm{mg} / \mathrm{kg}$ in .5 cc normal saline 15 minutes prior to central injection. Solutions of .25 scopolamine $\mathrm{HCl}$ were injected using the same parameters as arecoline. All Ringer-Locke's solution was buffered to physiological normalcy and all injection volumes were of such a size so as to minimize nonspecific effects such as pressure changes and ventricular distention. Injections were administered by a manually operated 705 Hamilton microliter syringe. Order of drug presentation was randomized across subjects. Dosages were based upon previous published reports (Beleslin and Samardzic, 1979) and preliminary studies in our laboratory.

\section{Apparatus}

Preliminary protocols to deterinine behavioral responses to stimulation were carried out in a $61 \mathrm{~cm} \times 61 \mathrm{~cm} \times 61 \mathrm{~cm}$ isolation chanber which has been described previously (Katz and Thomas, 1975).

To assess reflexive aspects of attack the subject was immobilized in a canvas feline restraint bag (Lentz Inc.) modified to allow free movement of either paw.

\section{Surgery}

Subjects were anesthetized via intraperitoneal injection of $35 \mathrm{mg} / \mathrm{kg}$ sodium pentobarbital (Nembutal). Each subject was stereotaxically implanted with twelve stainless steel electrodes . $24 \mathrm{~mm}$ in diameter insulated to the tip, and one stainless steel cannula $.8 \mathrm{~mm}$ in diameter. In addition, two indifferent electrodes were attached to stainless steel screws in the skull for purposes of monopolar stimulation. All electrodes were aimed at the lateral hypothalamic area and the perifornical area, and the cannula was aimed at the third ventricle. All placements were based on the atlas of Snider and Neiner (1964). Surgery was performed under aseptic conditions. At the close of surgery 150,000 units of Bicillin were administered intramuscularly.

\section{Experimental Procedure}

One week after surgery animals were tested for responding to stimulation. Initial testing was carried out in the isolation chamber. During initial testing and throughout the course of the experiments stimulation consisted of monophasic $1.0 \mathrm{msec}$ duration pulses, delivered through a $2 \mu \mathrm{F}$ capacitor in series with the animal to provide for reversal of current. Pulses were delivered 150 pulses per second in 10 second trains, with 120 to 150 second intervals between stimulations. All stimulation was delivered by a Grass SD-9 stimulator and monitored across a $100 \Omega$ resistor in series with the subject, on a 122-A Hewlett-Packard oscilloscope. Current levels were determined immediately prior to each stimulation by the presentation of a single $1.0 \mathrm{msec}$ pulse. Sites which yielded consistent quiet biting attack were selected for further testing. 
Based upon initial protocols, subjects were tested for reflexive behaviors. Three levels of current were employed: subthreshold, threshold, and suprathreshold. Threshold current was defined as the lowest level of stinulation in which at least one patterned reflex was elicited during two consecutive stimulations. Subthreshold current was defined as a current value .05 to $.1 \mathrm{ma}$ below threshold, and suprathreshold current was defined as a current value .05 to $.1 \mathrm{ma}$ above threshold. It should be noted that once current levels were specified for a given subject they remained constant for the remainder of testing.

Subjects were tested twice consecutively at each level of stimulation with order of stimulations randomized. Several current paraneters were employed in order to maximize any weak drug effects that might be confounded by either ceiling or floor effects. For example, potentiation that might be diminished or masked at threshold levels of stimulation might conceivably be demonstrable with subthreshold levels of stimulation. For similar reasons suprathreshold levels of stimulation might be the most sensitive test for potential inhibitory effects of drugs. Testing was carried out in two 30 to 45 minute sessions.

Subjects were tested for reflexes of biting, head turning, and pawing in the restraint bag already described. Subthreshold, threshold, and suprathreshold levels of stimulation were employed to assess each of three terminal reflexes elicited by proximal stimulation of the lipline, face and anterior paw surfaces of the subject contralateral to the site of stimulation. Light tactile stimulation using a standard $1 \times 1$ cm sponge rod was manually applied. Based upon two stimulations at each current level, a probability of response measure was obtained for each level of stimulation.

For a given drug three stages of testing were employed. These were: establishment of a set of baseline behaviors under the appropriate control injections; drug administration and subsequent testing of behaviors, and a post-drug recovery test, which served as the pre-drug baseline for subsequent drug testing. Post-drug testing occurred at least 24 hours after drug testing.

\section{Histology}

At the close of the experiment all cats were injected with an overdose of Nembutal, and perfused first with a solution of normal saline and subsequently with a solution of $10 \%$ formaldehyde in saline. Brains were removed, sliced in 40 micron sections, and examined, using a modification of Skinnner's photographic histologic technique (Skinner, 1971). All electrode placements that were used were in the lateral hypothalamic area, and all cannulae were in the third ventricle.

\section{Results}

All results are presented as mean and standard error. Statistical analysis was based upon within subjects comparisons using the t-test (Dixon and Massey, 1969) or, when the assumptions of a t-test could not be met, by randomization tests (Siegel, 1956).

Figure 1 presents arecoline effects upon reflexive biting. It may be seen there was significant potentiation of reflexive biting below and at threshold $t_{r b} s u b=5.57, t_{r b} t h r=2.64 ; d f=6$ in both cases).

Figure 2 presents similar data for reflexive pawing; reflexive pawing was potentiated both at and below threshold ( $t_{r p}$ sub $=5.57, t_{r p} t h r=3.24 ;$ $\mathrm{df}=6$ in both cases). Figure 3 presents data for reflexive head turning. It may be seen that arecoline induced an increase in head turning at 
subthreshold levels of stimulation. Since both predrug and drug scores have 0 variance, a t-test inay not be used in evaluating this increase. However a randomization test for paired data shows this increase to be significant beyond chance ( $p<.01$, two tailed randomization test, for paired data, $n=7$ ). Informal observations of drug stimulated cats indicated consistent (7/7) patterns of mydriasis and hissing during testing.

Results for scopolamine injection are presented in Figs. 4 through 6. Fig. 4 presents data on biting.

In addition there was a significant depression of pawing above threshold $(t$ rp $\sup =2.76, \mathrm{df}=7 ; \mathrm{Fig} .5)$. Fig. 6 presents data on head turning. No differences reach statistical significance on a two-tailed criterion. However, since Katz and Thomas (1975) and others have previously shown that systemically administered anticholinergic agents can block many aspects of predation the use of a 2-tailed test inight in fact be unduly restrictive. If this criterion is relaxed then suprathreshold scores are significanlty depressed $\left(t_{r p}\right.$ sup $\left.=2.0, d f=7\right)$. As in arecoline testing mydriasis was

consistently present in all cats after drug. Post-drug data are not presented separately; at no point did predrug-post-drug differences reach statistical significance.

\section{Discussion}

To date only limited data have been provided specifically examining the role of neurotransmitters in the elicitation and supression of patterned reflexes. The present findings support a cholinergic component and in addition anplify previous results from our laboratory (Katz and Thomas 1975; Katz, 1976) and the laboratories of others (Berntson and Leibowitz, 1973)

Since patterned reflexes associated both with predation and affective attack were elicited one possible interpretation of these results is that the cholinergic synapse plays a fundamental role as the final common path for expressing many forms of aggression, and that additional neurochemical control modifies and selects from a nonspecific aggressive substate to produce specific syndromes. It should also be realized with this in mind that normal interspecific aggression is characterized by aspects of both affective and predatory behavior (Leyhausen, 1973), and also that recent studies from Smith and Flynn (1979) make it evident that a central nervous system predatory substrate may directly support pawing behavior. Given the findings of both ethologists and physiologists the ascription predatory or affective significance to the reported behaviors cannot be unequivocal. This reflects intrinsic properties of, and commonalities of, aggressive behaviors. Indeed it may be more useful to accept such overlap as necessary, and to reject post hoc ascription in the absence of tests involving ecologically meaningful goal objects.

\section{Conclusion}

In conclusion, the present findings confirm a role for a inuscariniccholinergic system in the control of aggression. This role includes the normal facilitation of several species of patterned reflexes. Additionally it may include other as yet unspecified behaviors.

\section{Acknowledgments}

Portions of this work in somewhat modified form comprised two experiments from a doctoral thesis submitted to, and accepted by, the Departinent of 


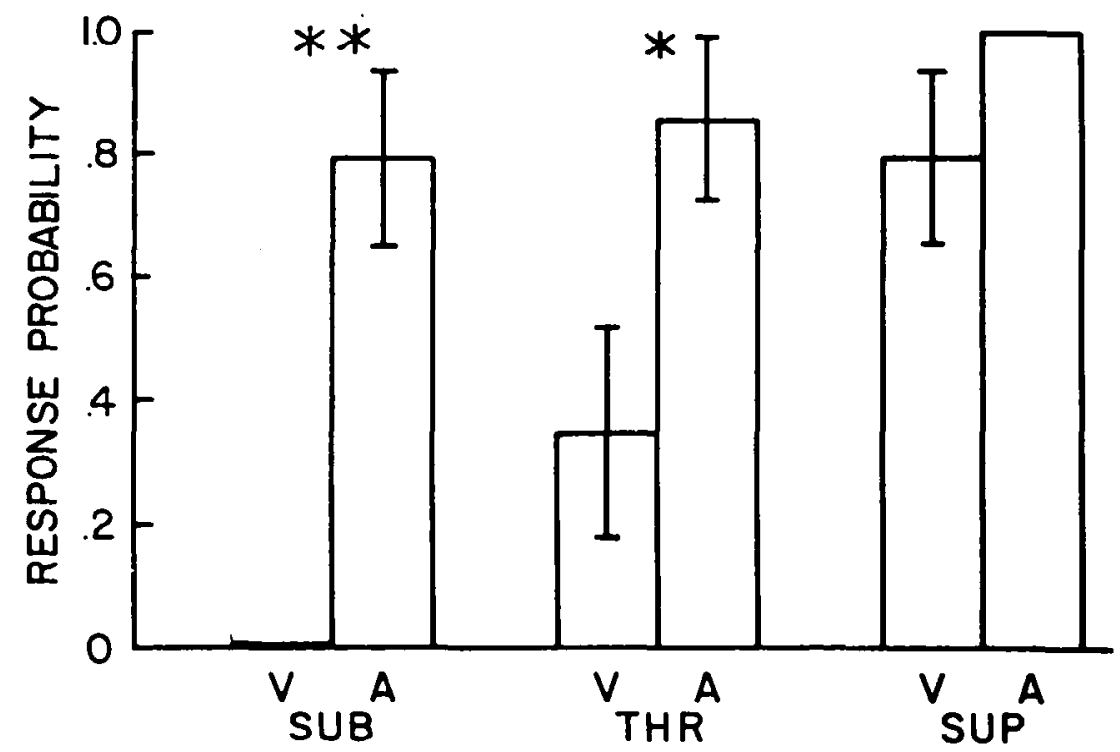

Fig. 1. Effects of arecoline upon biting. V=vehicle; A=arecoline; sub=subthreshold; thr=threshold; sup=suprathreshold, * $p<.05 \underline{t}$ test for paired observations; **p<.01 employing the same test.

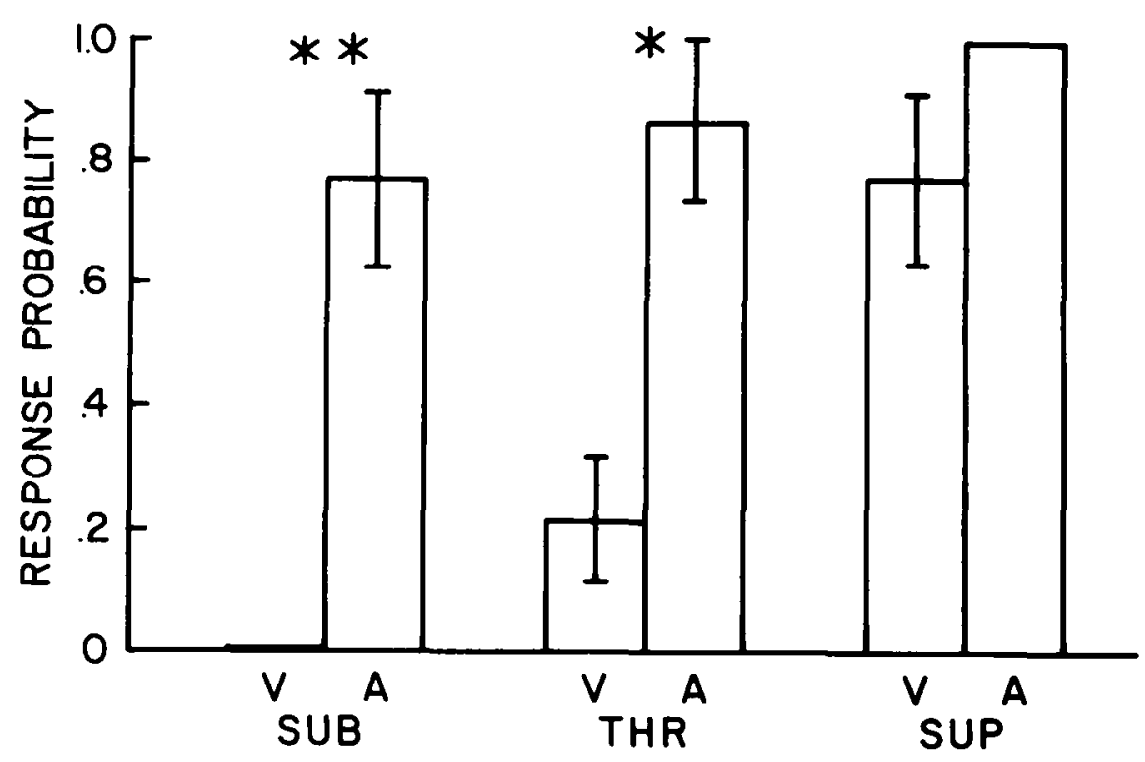

Fig. 2. Effects of arecoline upon pawing. V=vehicle; $A=$ arecoline; sub=subthreshold; thr=threshold; sup=suprathreshold, * $p<.05, \underline{t}$ test for paired observations; ** $p<.01$ based upon the same test. 


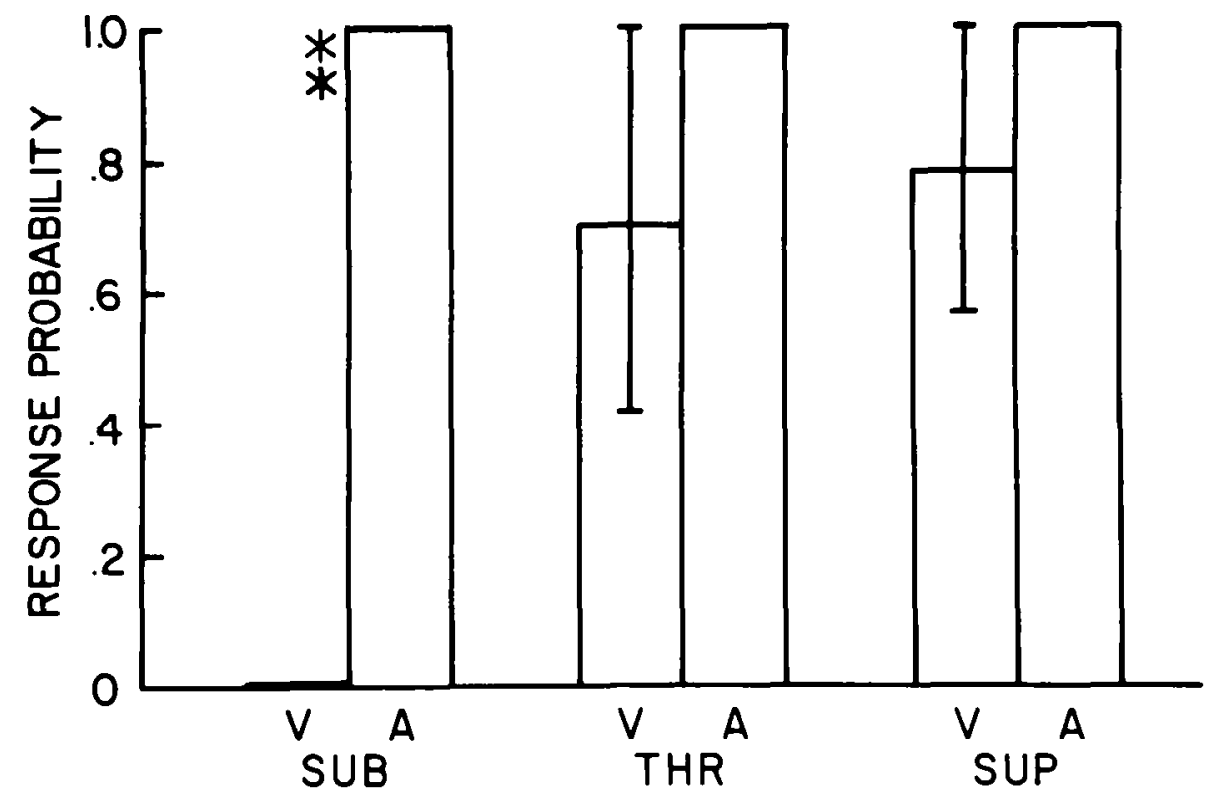

Fig. 3. Effects of arecoline upon turning. V=vehicle; $A=\operatorname{arecol} i n e$; ** p<.01, randomization test.

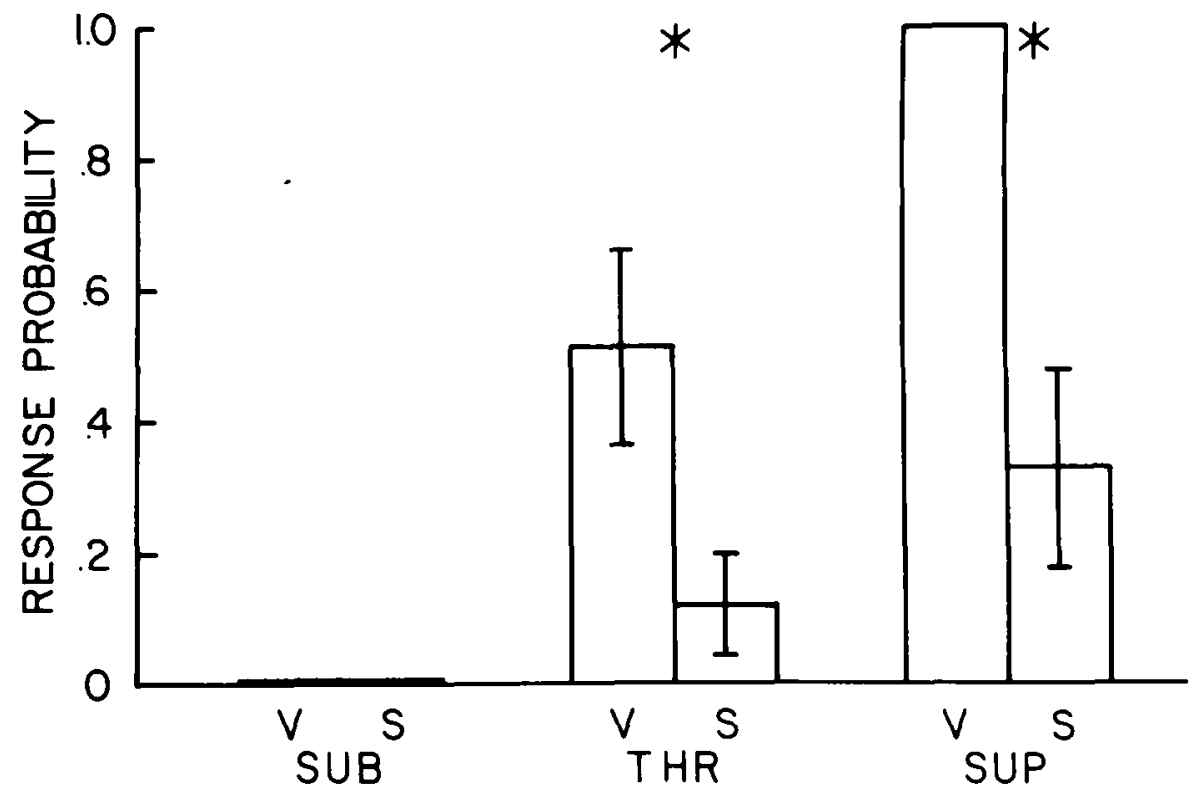

Fig. 4. Effects of scopolamine upon biting. V=vehicle; $S=s c o p o l a m i n e ; s u b=s u b t h r e s h o l d ; ~ t h r=t h r e s h o l d ; ~ s u p=s u p r a t h r e s h o l d$, * $p<.05$, t test for paired observations. Scopolamine depressed biting both at and above threshold ( $t_{r b}$ thr $=3.24, t_{r b} \sup =3.21$, $d f=7$ for both cases). 


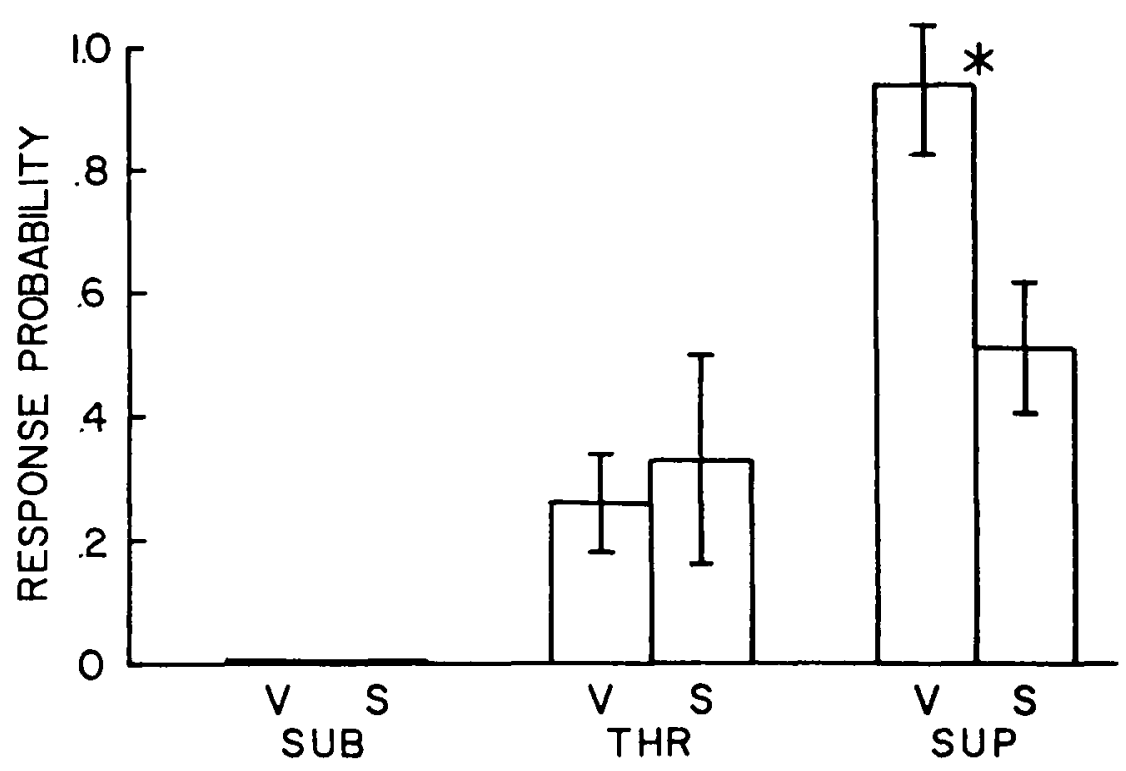

Fig. 5. Effect of scopolamine upon pawing. V=vehicle; $S=$ scopolamine; $s u b=$ subtreshold, * $p<.05$, $t$ test for paired observations.

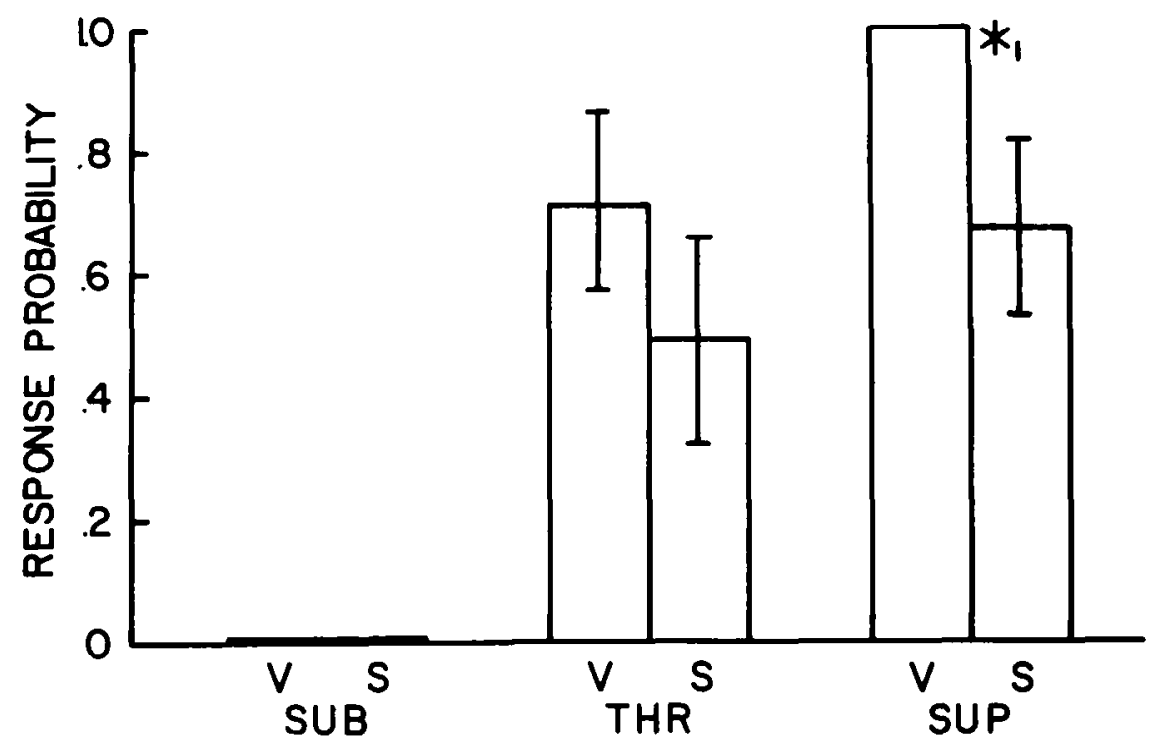

Fig. 6. Effects of scopolamine upon turning $V=v e h i c l e ;$ $\mathrm{S}=$ scopolamine; sub=subthreshold; thr=threshold; sup=suprathreshold, * $p<.05,1$ tailed $t$ test for paired observations. 
Psychology of Bryn Mawr College. The research was direoted by Drs. Larry Stein and Earl Thomas, and thesis committee members include Drs. R.C. Gonzales, C. McCauley, M. Yarczower, H.S. Hoffinan, and E. Pepitone (as outside chair). I am grateful to all the above, particularly to Drs. Stein, Thomas, and Hoffman for their comments upon the work, corrections, emendations, and support. The notion of a general cholinergic substance for aggression was developed in conversation with Howard Hoffman. Financial support in part was from grant MIA 15946 to Earl Thonas.

\section{References}

BELESLIN, D.B. and SAMERDZIC, R. (1979). Comparative study of aggressive behavior after injection of cholinomimetics, anticholinesterases, nicotinic and insscarinic ganglionic stimulants into the cerebral ventricles of conscious cats:. Failure of nicotinic drugs to evoke aggression. Psychopharmacology, 60: 147-54.

BERNTSON, G.G, and LEIBOWITZ, S.F. (1973). Biting attack in cats: Evidence for central muscarinic mediation. Brain Res., 51:366-370.

BERNTSON, G.G. and MICCO, D.J. (1976). Organization of brainstem behavioral systems. Brain Res. Bull., 1:471-483.

DIXON, W. and MASSEY, F. (1969). Introduction to statistical analysis. McGraw Hill, New York.

FLYNN, J.P. (1967). The neural basic aggression in cats. In: Neurophysiology and Emotion, D.C. Glass (ed.). pp. 40-59. Rockefeller University
Press, New York.

FLYNN, J.P. (1972). Patterning mechanisms, patterned reflexes and attack behavior in cat. Nebraska Symposium on Motivation 1972, J.K. Cole and D.P. Jensen (eds.). pp. 125-154. University of Nebraska Press, Lincoln.

KATZ, R.J. (1976). Effects of the cholinomimetic drug arecoline upon aggression: Intra- vs Inter-specific allocation of attack. Aggressive Behavior, 2: 205-212.

KATZ, R.J. and THOMAS, E. (1975). Effects of scopolamine and methyl paratyrosine upon predatory attack in cats. Psychopharmacologia (Berl), 42: $153-157$.

LE $\overline{Y H A U S E N, ~ P . ~(1973) . ~ V e r h a l t e n s t u d i e n ~ a n ~ K a t z e n . ~ 3 r d ~ e d . ~ P a u l-P a r e y, ~}$ Berlin.

SIEGEL, S. (1956). Non parametric statistics for the behavioral sciences. MoGraw Hill, New York.

SKINNER, J.E. (1971). Neuroscience: A Laboratory Manual. Saunders, Phil adel phia.

SMITH, D.A. and FLYNN, J.P. (1979). Afferent projections related to attack sites in the pontine tegmentum. Brain Res., 164:103-120.

SNIDER, R.S. and NEIMER, W.T. (1964). A stereotaxic atlas of the cat brain. University of Chicago Press, Chicago.

Inquiries and reprint requests should be addressed to:

Dr. R.J.Katz

Mental Health Research Institute, Department of Psychiatry

University of Michigan Medical Center, Ann Arbor, MI 48109, USA 